\title{
Circular Array Antenna for UAV-UAV Communications
}

\author{
Xiaoliang Sun , Rodrigo Blázquez-García , Alejandro García-Tejero , José Manuel Fernández-González , \\ Mateo Burgos-García, Manuel Sierra-Castañer
}

\begin{abstract}
Nowadays, the communications links limit the autonomy of unmanned aerial vehicles (UAV). In this paper, a multichannel long-range communication link for UAVs with high quality of service, moderate bandwidth and affordable cost is proposed. This link is deployed by using a second UAV as a communications relay and it provides bidirectional coverage for telemetry and telecommand and a high-capacity downlink for video. Our highly compact system can be installed on medium-sized UAVs for air-air links in order to offer greater flight autonomy. This system is based on an active circular array of linearly polarized circular patch antennas which are selectively activated depending on the desired direction of the beam.
\end{abstract}

Index Terms-air link, circular patch, conformal array antenna, RF front end, UAV.

\section{INTRODUCTION}

The autonomy of unmanned aerial vehicles (UAV) is currently limited by the communications links used for sending telecommands and the data captured by the sensors of the airborne platform. Using an S, C or K band line-ofsight radio link is the most common strategy. However, these links imply reduced autonomies which are highly dependent on the terrain orography. A second possibility is the usage of a satellite connection. In this case, either a very expensive space segment or an Iridium-like system is used. The former is not economically feasible for small and medium-sized UAVs whereas the latter has communication outages and an insufficient quality of service for this application. Therefore, the current state of UAV communications is that data links have short ranges, high costs, low capacity or low reliability.

In this paper, a multichannel long-range communication link with high quality of service, moderate bandwidth and affordable cost is proposed. The objective is to achieve a 70 $\mathrm{km}$ range. As shown in Fig. 1, this link is deployed by using a second UAV as a communications relay. This UAV is kept close to the ground station to guarantee the line-of-sight connectivity, and at a sufficient altitude to avoid terrain obstacles that can block the link between the UAVs.

The communications system has to provide bidirectional coverage for telemetry and telecommand, and a highcapacity downlink for video or equivalent sensors.

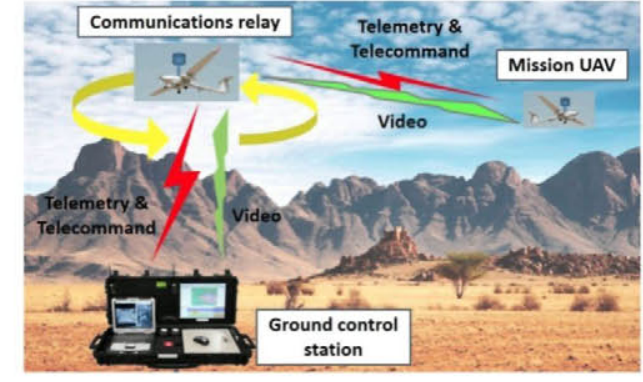

Fig. 1. Long-range UAV-relay system.

The key point of the aerial link is the radiating system. The usage of a narrow beam antenna in both UAVs increases significantly the range of the system. The main problem that arises is to keep both antennas pointed for any relative position and any flight attitude of the UAVs. Besides, commercial-off-the-shelf (COTS) devices for radio links at C band $(5 \mathrm{GHz})$ are going to be used because the imminent normalization of this band will promote the development of equipment with good price-performance ratio.

This solution will provide greater flight autonomy for UAVs than the current ones. Several advances have been achieved in this regard, but none of them with the extent addressed in this innovative system. The application of smart antennas with tracking systems for ground stations [1] has been developed during the last few years, and they are in operation for medium-sized and large systems. However, there hardly exist any similar systems for airborne platforms.

A communications system with smart antennas for UAVs is analysed theoretically in [2], but it is not physically implemented due to the high complexity of the design. A solution that has been implemented is shown in [3] as an emerging technology identified by the European Space Agency. However, the limitations of this system make it unfeasible for the application depicted in Fig. 1. It can be applied only to air-ground links of UAVs with high attitude stability and it has a limited pointing margin.

Our proposed system is highly compact and can be installed on medium-sized UAVs for air-air links with medium gain antennas. In addition, it can be pointed over a $360^{\circ}$ azimuth range for any attitude of the UAVs. 


\section{ANTENNA SYSTEM DESIGN}

\section{A. Antenna element}

The designed transmitter and receiver antennas are composed of a circular array of eight elements, whose specifications are shown in Table I. The microstrip vertically polarized circular patch antenna is used as the basic radiating element due to its advantages of small size, low profile, simple geometry and low cost [4]. Fig. 2 shows the geometry of one element. It consists of one circular patch, whose radius is $13.7 \mathrm{~mm}$, a circular feeding disk stacked between three substrate layers and a rectangular ground plane. The lengths and widths of three substrates and the ground plane are all $58 \times 30 \mathrm{~mm}$. As shown in Fig. 3, the top substrate is a $0.254 \mathrm{~mm}$-thick FR4 followed by a $3 \mathrm{~mm}$-thick FOAM. The following layers staked at the bottom of the FOAM are respectively a $4 \mathrm{~mm}$-thick aluminium and a $0.5 \mathrm{~mm}$-thick Taconic $5 \mathrm{~A}$, whose relative permittivity $\left(\varepsilon_{R}\right)$ is 2.17 . On this last substrate, the feeding and T/R circuits are mounted.

Fig. 2 shows that the copper feeding disc is connected to the centre conductor of a coaxial cable, which is located at a distance of $5.7 \mathrm{~mm}$ from the centre of the circular patch. Besides, two planes made of aluminium are used at the top and bottom to reduce the undesirable electromagnetic interference from the other array and improve the isolation between receiver and transmitter. Their length is $20.5 \mathrm{~mm}$.

TABLE I. SPECIFICATIONS OF THE ANTENNA ELEMENT

\begin{tabular}{|l|l|}
\hline Working Band & $5.03 \mathrm{GHz}-5.091 \mathrm{GHz}$ \\
\hline Gain & $>6 \mathrm{~dB}$ \\
\hline Beamwidth in elevation & $>50^{\circ}$ \\
\hline
\end{tabular}
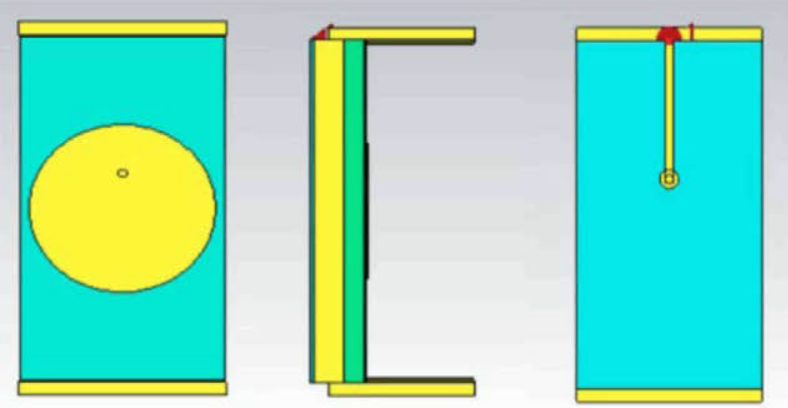

Fig. 2. Geometry of the proposed patch antenna.

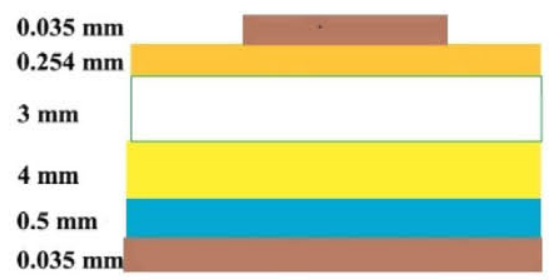

Copper

Fr4 $\mathrm{Er}=4.3$

Foam Er=1.07

Groundplane (Aluminum)

Taconic TLY-5A Er=2.17 Copper

Fig. 3. Structure of the patch antenna.

The antenna operates at $5.065 \mathrm{GHz}$. One radiating element has been simulated in CST to optimize the return loss. As presented in Fig. 4, a bandwidth of $110 \mathrm{MHz}$, which is enough to cover the working band, is achieved. The vertical plane radiation pattern of one element is represented in Fig. 5, from which it can be observed that the HPBW (Half Power Beam Width) in elevation is about $50.7^{\circ}$, which satisfies our requirement for one patch antenna.

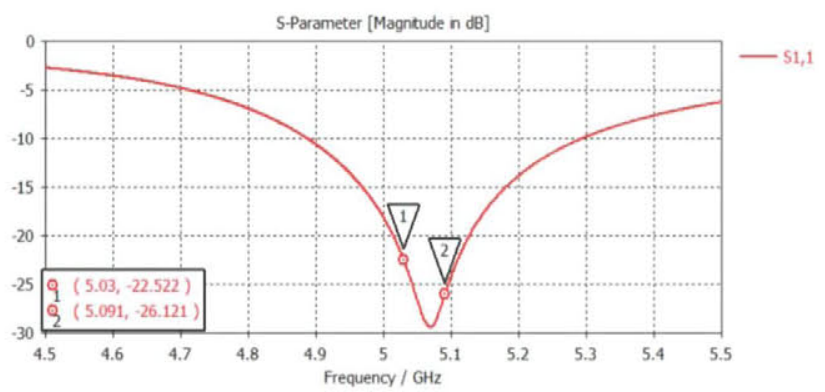

Fig. 4. Simulated $\mathrm{S}_{11}$ parameter for an array element.

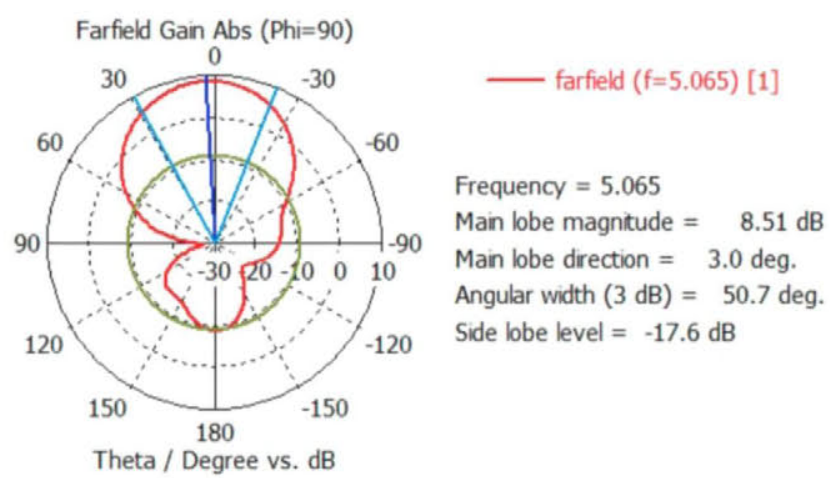

Fig. 5. Simulated radiating pattern for an element (vertical plane).

Fig. 6 represents the geometry of a $2 \times 2$ element configuration in including both transmitter and receiver antennas, the angle between transmitters or receivers is $45^{\circ}$. To get a better analysis, every element is given a number. The transmitter and receiver antennas are separated by aluminium material.

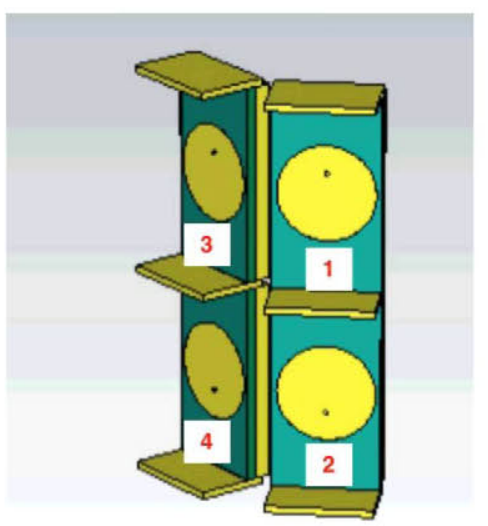

Fig. 6. Geometry of $2 \times 2$ elements for transmitter (the upper two) and receiver (the lower two).

The optimized S-parameter simulation results, when only one element is activated, are depicted in Fig. 7 in order to show the isolation between transmitter and receiver 
elements. In the considered bandwidth, a return loss for each individual element better than $20 \mathrm{~dB}$ and an isolation better than $35 \mathrm{~dB}$ between transmitting and receiving elements are achieved. The radiation pattern normalized to the total input power is shown in Fig. 8 for both horizontal and vertical planes. Because there are eight elements and we have eight combinations of two elements, each combination horizontal should cover $45^{\circ}$. The HPBW is $46.1^{\circ}$ in horizontal plane and $52.2^{\circ}$ in vertical plane, which are suitable for both dimensions.

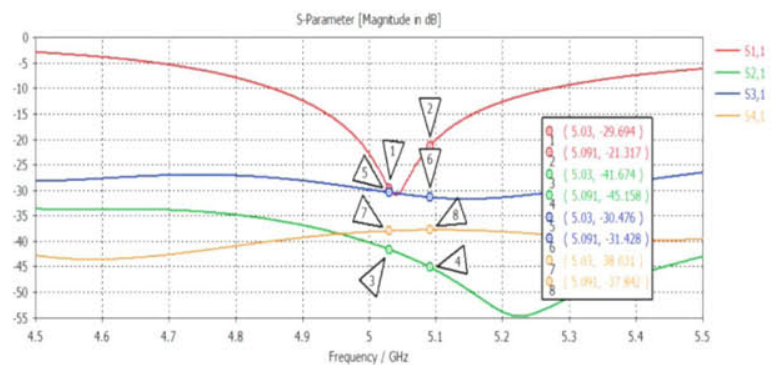

Fig. 7. Simulated $\mathrm{S}$ parameters for $2 \times 2$ configuration when one element is activated.
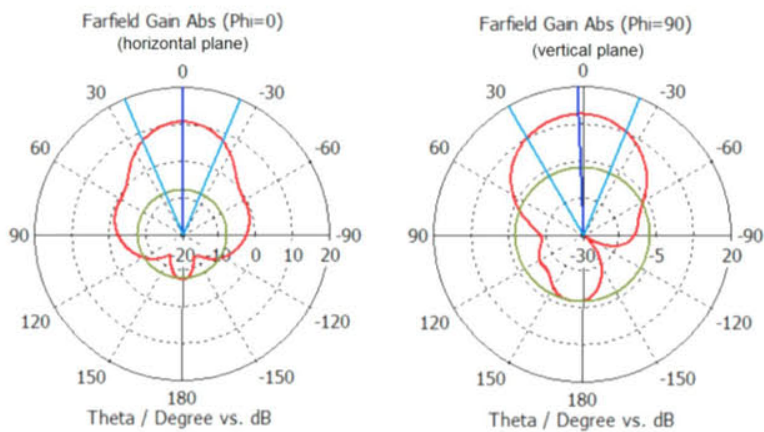

Fig. 8. Simulated horizontal and vertical plane radiation patterns for the $2 \times 2$ configuration.

The designed antenna array structure is shown in Fig. 9. It is a circular array with 16 elements, the 8 upper elements are used for transmission and the lower ones are used for reception.

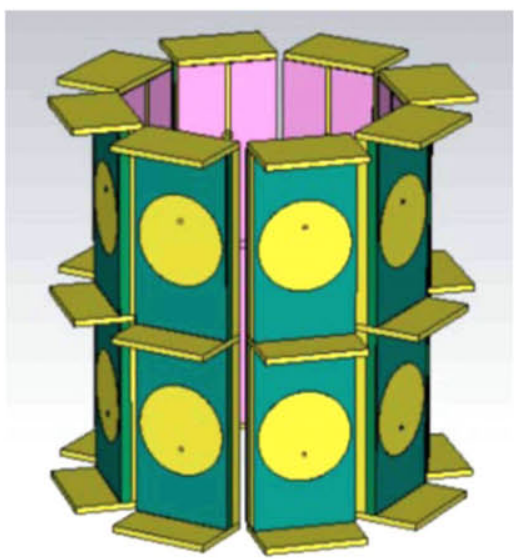

Fig. 9. Complete architecture of the designed antenna array.

\section{Final Design AND Results}

In this section, the final design and measurement results are presented. Fig. 10 shows one first prototype of the design implemented during this work.
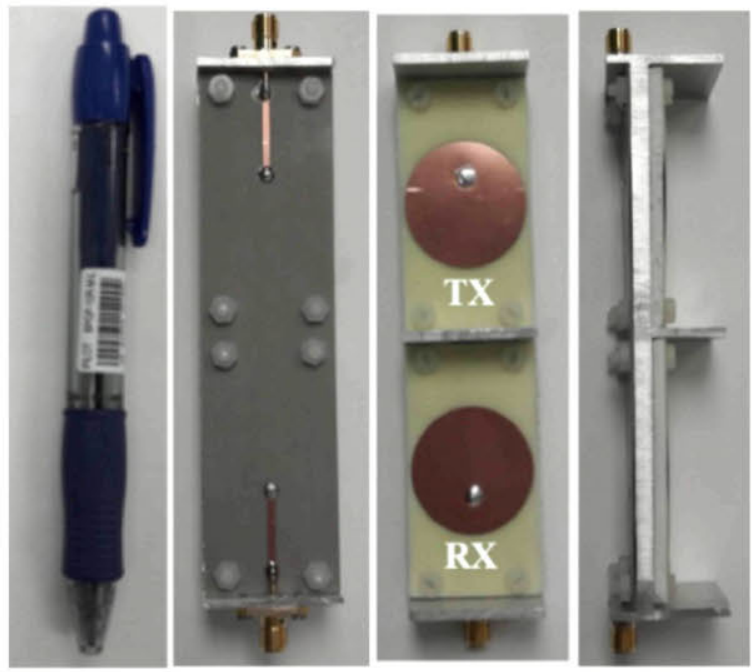

Fig. 10. Prototype of the element array.

Fig. 11 presents the comparison between measured and simulated S-parameters. The frequency of the minimum measured reflection coefficient has moved in comparison with the simulated result in CST because we used a substrate with $0.2 \mathrm{~mm}$ of thickness instead of $0.254 \mathrm{~mm}$ but in the working band the measured reflection coefficient is below $-20 \mathrm{~dB}$. The measured and simulated isolation between transmitter and receiver is better than $-40 \mathrm{~dB}$. Fig. 12 shows the measurement setup of the combination of two elements in azimuth in the anechoic chamber of Universidad Politécnica de Madrid, and Fig. 13 presents the measured and simulated radiation pattern at centre frequency. The measurements and simulation fulfill the specifications.

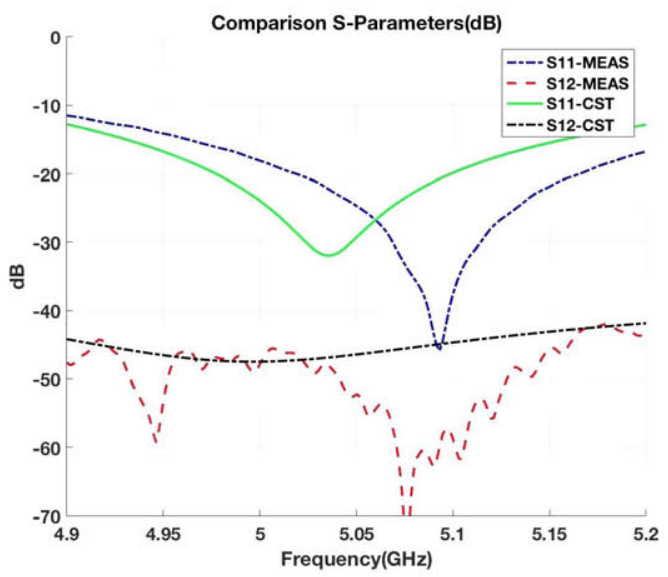

Fig. 11. Comparison between simulated and measured S-parameters. 


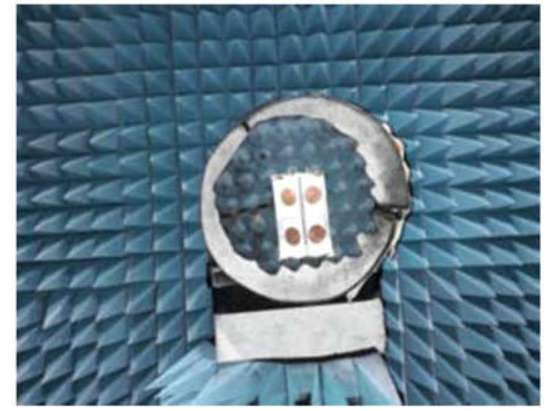

Fig. 12. Measurement setup in the anechoic chamber of Universidad Politécnica de Madrid.

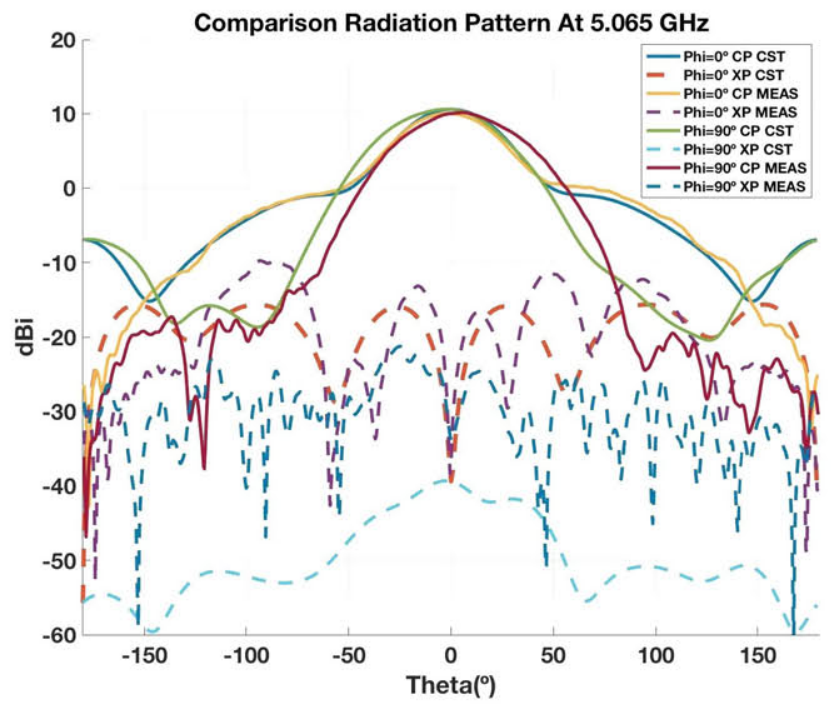

Fig. 13. Comparison of simulated and measured radiation pattern at centre frequency $(f=5.065 \mathrm{GHz})$.

\section{CONCLUSIONS}

This paper has presented the design of a circular array antenna for an UAV communications system to provide airair links and extend the autonomy of current medium-sized UAVs by using a second UAV as a relay. The antenna module consists of two circular arrays, one for reception and the other for transmission, with eight vertically polarized circular patches that are selectively activated depending on the desired direction of the beam. The manufactured antenna has been measured, achieving an antenna gain of $10 \mathrm{~dB}$, a reflection coefficient below $-20 \mathrm{~dB}$ and an isolation between $\mathrm{Tx}$ and $\mathrm{Rx}$ antennas better than $-40 \mathrm{~dB}$ in the working band.

\section{ACKNOWLEDGMENTS}

The authors would like to thank Unmanned Solutions, S.A. for its support to this work. The authors also want to acknowledge the Madrid Region Government for financing the project S2013/ICE-3000 SPADERADAR-CM and the Spanish Government, Ministry of Economy, National Program of Research, Development and Innovation for the support of the projects ENABLING5G "Enabling Innovative Radio Technologies for 5G networks" (Project number
TEC2014-55735-C3-1-R) and "Array Digital en Recepción para Radares FMCW de Vigilancia Superficial" (Project number TEC2014-53815-R).

Simulations done in this work have been performed using CST Microwave Studio Suite 2016 under a cooperation agreement between Computer Simulation Technology (CST) and Technical University of Madrid.

\section{REFERENCES}

[1] S. Jenvey, J. Gustafsson and F. Henriksson, "A portable monopulse tracking antenna for UAV communications," 22nd International Unmanned Air Vehicle Systems Conference, April 2007, pp. 1-8.

[2] J. C. Porcello, "Designing and implementing multibeam smart antennas for high bandwidth UAV communications using FPGAs," 2013 IEEE Aerospace Conference, March 2013, pp. 1-12.

[3] DAAA - Drone Active Antenna Array [online]. Retrieved from http://www.esa-tec.eu/space-technologies/from-space/daaa-droneactive-antenna-array/

[4] R. Garg, P. Barthia, I. Bahl and A. Ittipiboon, Micostrip Antenna Design Handbook. Norwood, MA: Artech House, 2001.

[5] C. A. Balanis, Antenna Theory, Analysis and Design. Hoboken, NJ: John Wiley \& Sons, Inc., 2005. 